\title{
Melanoma maligno gastrointestinal de origen primario desconocido: reporte de caso
}

\section{A case report of malignant gastrointestinal melanoma of unknown primary origin}

\author{
Sandra Patricia Cifuentes A. ${ }^{1 *}$, Sandra Lorena Santacruz R. ${ }^{2}$
}

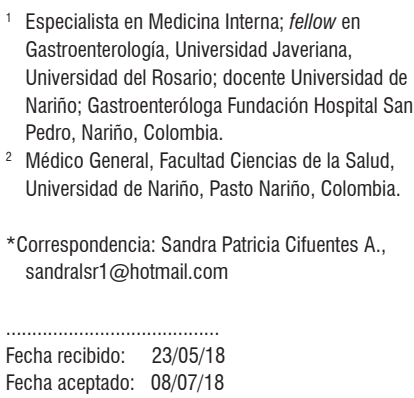

\begin{abstract}
Resumen
Una de las características inusuales del melanoma cutáneo es la capacidad de metastatizar en el intestino delgado. A menudo se diagnostica durante la autopsia, en la cual se ha encontrado depósitos metastásicos en el $50 \%-60 \%$ de los casos, pero menos del $2 \%$ al $4 \%$ de los pacientes con diagnóstico de melanoma tiene metástasis gastrointestinal (GI) durante el curso de la enfermedad y entre el $4 \%-9 \%$ de casos de melanoma GI tiene un primario desconocido. La rápida identificación y resección del melanoma en el tracto digestivo podría mejorar la tasa de supervivencia en aquellos pacientes en los que se presentan complicaciones, como obstrucción intestinal.

Se presenta un caso clínico de melanoma gastrointestinal de origen primario desconocido. El porcentaje de este tipo de casos es poco frecuente. La paciente presentó cuadro clínico de náuseas, hiporexia, epigastralgia, fatiga, parestesias en región dorsal derecha y disminución de 9 kilogramos en 3 semanas. Le realizaron tomografía computarizada (TAC) de abdomen contrastado, que reportó 3 lesiones en hígado de predominio isodenso en el parénquima, con algunas áreas de menor densidad al interior, localizadas en los segmentos 2, 5, 7 y 8 del hígado al que se le realizó la biopsia. Se llevó a cabo endoscopia de vías digestivas altas con biopsia de 2 lesiones hiperpigmentadas en la segunda porción de duodeno. El examen histopatológico de las biopsias evidenció melanoma maligno. Se excluyeron todas las localizaciones típicas de melanoma primario durante el procedimiento diagnóstico.
\end{abstract}

\section{Palabras clave}

Melanoma, metástasis, neoplasias primarias desconocidas, tracto gastrointestinal.

\begin{abstract}
One of the unusual characteristics of cutaneous melanoma is its ability to metastasize in the small intestine. It is often diagnosed during autopsies of cutaneous melanoma patients. Metastatic deposits have been found in $50 \%$ to $60 \%$ of these autopsies, but less than $2 \%$ to $4 \%$ of patients diagnosed with melanoma have gastrointestinal metastasis during the course of the disease. Between $4 \%$ and $9 \%$ of gastrointestinal melanoma cases have unknown primary tumors.

Rapid identification and resection of melanoma in the digestive tract could improve the patient survival rate and prevent complications such as intestinal obstructions from occurring. We present a rare clinical case of gastrointestinal melanoma of unknown primary origin. The patient had a clinical picture of nausea, hyporexia, epigastralgia, fatigue, paresthesias in the right dorsal region and had lost nine kilograms in three weeks. An abdominal CT scan showed three predominantly isodense liver lesions in the parenchyma, with some areas of lower density located in segments 2,5,7 and 8 of the liver. These were biopsied. Upper digestive tract endoscopy took biopsy samples of two hyperpigmented lesions in the second portion of the duodenum. Histopathological examination showed malignant melanoma. All typical locations of primary melanoma were excluded during the diagnostic procedure.
\end{abstract}

\section{Keywords}

Melanoma, metastasis, unknown primary neoplasms, gastrointestinal tract. 


\section{INTRODUCCIÓN}

El melanoma maligno (MM) es un tumor que se origina en las células neuroectodérmicas, formado a partir de los melanocitos. El foco primario de este cáncer generalmente se diagnostica en piel, globo ocular o ano. El melanoma de origen primario desconocido representa el $1 \%$ a $8 \%$ de todos los casos (1-3). Por lo general, se diagnostica cuando los pacientes presentan síntomas clínicos en forma de metástasis. Con el fin de hacer un diagnóstico definitivo de melanoma de origen primario desconocido es necesario confirmar la presencia de metástasis histopatológica y excluir todos los lugares posibles de foco primario. Hay varias teorías que tratan de explicar la etiología del melanoma primario desconocido $(2,3)$. La primera es una regresión espontánea del foco primario como un efecto del sistema inmune y la otra es una transformación maligna de melanocitos individuales, que entran en un ganglio linfático sin formar un foco dentro de cualquier órgano $(2,3)$. El comportamiento biológico del tumor es similar a los casos con ubicación conocida del foco primario (4).

En la clínica, los melanomas malignos gastrointestinales son relativamente raros y frecuentemente metastatizan de primarios cutáneos (5). La incidencia de metástasis gastrointestinal de origen primario desconocido se encuentra entre $4 \%$ a $9 \%$ de los casos (6). Sin embargo, los melanomas primarios también pueden originarse de ciertas regiones en el tracto gastrointestinal (GIT), incluido el esófago, intestino delgado, recto y ano (5). El intestino delgado tiene la mayor predilección para el desarrollo de melanoma metastásico debido a su abundante irrigación sanguínea. El anorrecto es el sitio más común para melanoma primario GIT debido a la presencia de melanocitos $(7,8)$.

Esta es una neoplasia compleja y heterogénea, cuyas tasas de incidencia varían entre géneros, edad, grupos étnicos y región. Anualmente se diagnostican a nivel mundial aproximadamente 200000 nuevos casos de MM y 46500 muertes por esta causa (9). La tasa mundial de incidencia aproximada de MM fue de 2,5/100 000 en hombres y 2,6/100 000 en mujeres en el año 2002 (10). En las últimas 4 décadas se ha observado un incremento continuo de la incidencia del MM a nivel mundial, principalmente en regiones con poblaciones blancas; en Latinoamérica, los datos son escasos (9). Se conocen algunos reportes previos de la incidencia estimada en Colombia, si bien no se cuenta con un sistema de registro único del cáncer que permita establecer datos generales en la población colombiana, el Registro Poblacional del Cáncer de Cali es el único en el país que ha permitido el cálculo de la incidencia para una región específica $(11,12)$. Dicho registro ha evidenciado un ligero incremento en la tasa cruda de incidencia del MM, siendo de 1,6 en 1962-1966 y de 3,5 en 2003-2007 para hombres; en mujeres fue de 1 en 1962-1996 y de 3,2 en 2003-2007 (9).

Se presenta un caso clínico de melanoma metastásico a intestino delgado e hígado de origen primario desconocido. Como se describió anteriormente, el porcentaje de este tipo de casos es poco frecuente.

\section{REPORTE DE CASO}

Paciente femenina de 46 años, quien consultó al servicio de urgencias de la Fundación Hospital San Pedro por presentar náuseas, hiporexia, epigastralgia, parestesias en región dorsal derecha y disminución de $9 \mathrm{~kg}$ en 3 semanas. Como antecedentes patológicos personales refiere gastritis crónica. Dentro de los antecedentes patológicos familiares, su hermana murió de cáncer de pulmón y su padre tiene antecedente de cáncer de próstata. En cuanto a antecedentes quirúrgicos, hace 2 años tuvo dos cirugías de la retina del ojo izquierdo. $\mathrm{Al}$ examen físico fue hemodinámicamente estable, álgica, asténica, adinámica, deshidratada y con amaurosis en el ojo izquierdo. Abdomen levemente distendido, ruidos hidroaéreos positivos, dolor a la palpación de cuadrante superior derecho, sin signos de irritación peritoneal. En la piel tiene 2 lesiones pediculadas en antebrazo izquierdo y región lumbar derecha, respectivamente; el resto de la piel estaba sin lesiones sugestivas de melanoma.

Se le realizan paraclínicos, con cuadro hemático dentro de parámetros normales, PCR elevada: $15 \mathrm{mg} / \mathrm{dL}$, electrólitos, función renal, glucemia y tiempos de coagulación dentro de los valores normales. Ecografía de hígado y vías biliares que reportó múltiples lesiones focales de posible origen metastásico (Figura 1).

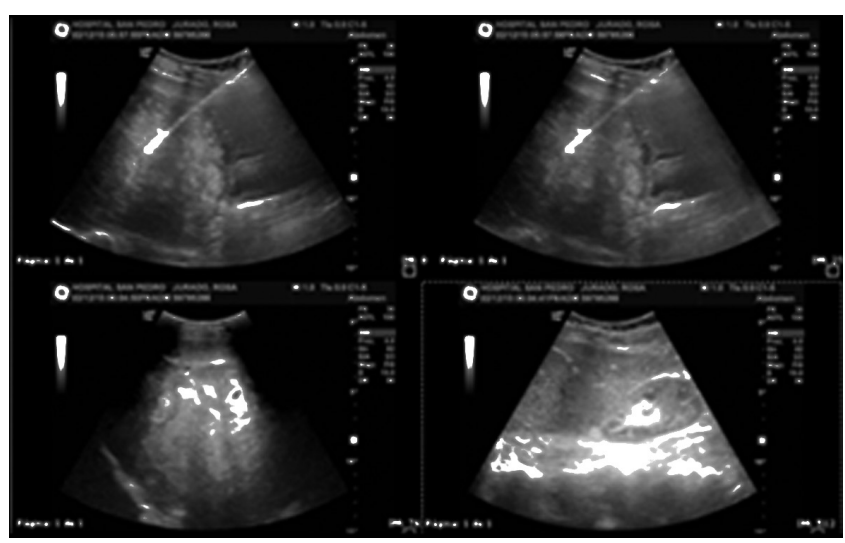

Figura 1. Ecografía de hígado y vías biliares. Múltiples lesiones focales de posible origen metastásico.

Se tomó TAC de abdomen contrastado y se encontró hígado de tamaño normal con contorno lobulado y ecogenicidad heterogénea. Se observaron 3 lesiones de pre- 
dominio isodenso con el parénquima, con algunas áreas de menor densidad al interior, localizada de mayor tamaño en los segmentos 5, 7 y 8, que medían $149 \times 128 \mathrm{~mm}$; y otras 2 lesiones hacia los segmentos 2 y 7 , con diámetros de 51 y $57 \mathrm{~mm}$; hallazgos que sugieren cambios por proceso neoplásico. El resto de los órganos abdominales se encontraban sin alteraciones (Figura 2).

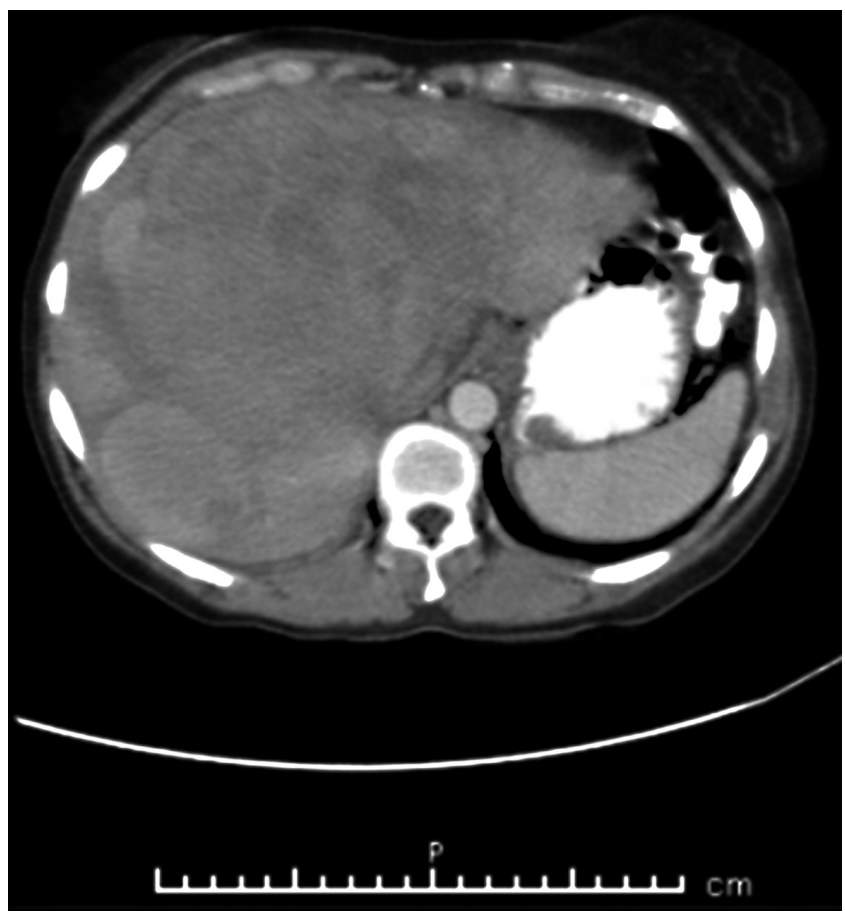

Figura 2. TAC de abdomen contrastado. Hígado con ecogenicidad heterogénea, en el que se observa 3 lesiones de predominio isodenso, con algunas áreas de menor densidad al interior, localizadas en los segmentos 5,7 y 8 y otras 2 lesiones hacia los segmentos 2 y 7 .

También se le realizó endoscopia de vías digestivas altas. Los hallazgos fueron gastritis eritematosa antral y dos lesiones hiperpigmentadas de $5 \mathrm{~mm}$ en segunda porción del duodeno (Figura 3), de las cuales se tomaron biopsias, cuyo resultado es de tumoración maligna, constituida por proliferación de células pleomórficas con núcleos hipercromáticos, nucléolo prominente y abundante pigmento melánico, se distribuyen en forma difusa en todo el espesor de la mucosa.

El diagnóstico histopatológico fue melanoma maligno. Se tomó biopsia percutánea de masa hepática, en la que se identificó tumoración maligna, constituida por proliferación de células pleomórficas con núcleos hipercromáticos, nucléolo prominente y abundante pigmento melánico. Se disponen en nidos y masas sólidas. Luego, la paciente ingresa por segunda vez al servicio de urgencias por los síntomas antes mencionado y con nuevos síntomas: coluria y hematoquecia. Se le tomaron paraclínicos con cuadro hemático de hemoglobina ligeramente disminuida 11,9; enzimas hepáticas ligeramente elevadas, TGO: 68, TGP: 54 , tiempos de coagulación e INR dentro de parámetros normales. Se le realizo colonoscopia total, la cual reportó hemorroides internas grado II, resto de colonoscopia total normal. Se obtuvo previo consentimiento informado para la elaboración del reporte de caso. La paciente actualmente se encuentra en quimioterapia paliativa y en manejo por medicina del dolor.

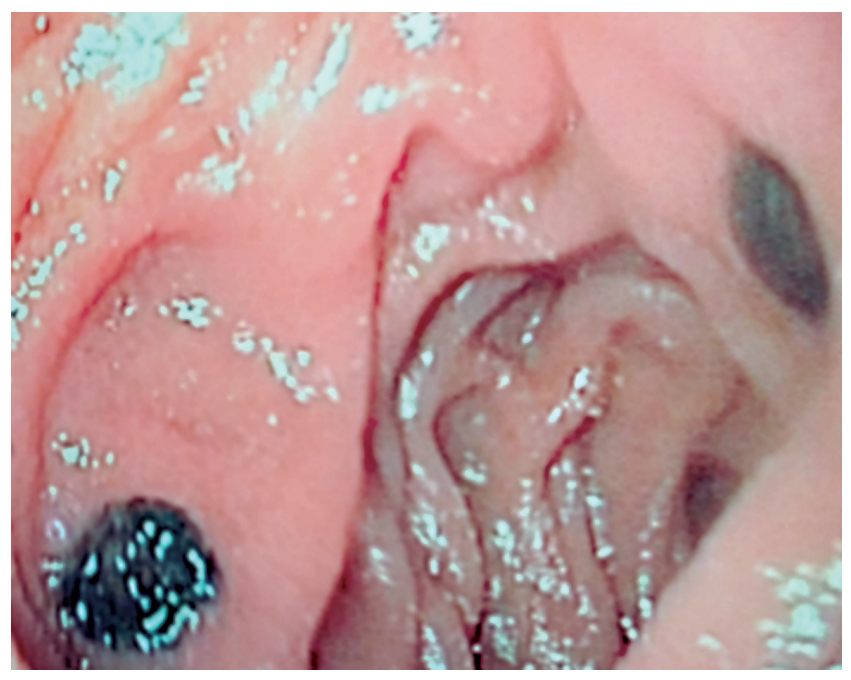

Figura 3. Endoscopia de vías digestivas altas. Duodeno segunda porción. Se observa en el bulbo 2 lesiones hiperpigmentadas de $5 \mathrm{~mm}$.

\section{DISCUSIÓN}

El melanoma maligno es un tumor que se presenta principalmente en la piel, con menos frecuencia en el ojo (capas coroides), leptomeninges, cavidad oral, mucosa nasal, faringe, esófago, bronquios, mucosa vaginal o anorrectal y uñas (11-13). En autopsias realizadas se ha informado metástasis GI en 50\%-60\% de los casos, pero solo 2\%-4\% de los pacientes con melanoma se diagnostica con metástasis GI durante el curso de su enfermedad. El melanoma primario en pacientes con metástasis GI se encuentra normalmente en las extremidades (15\%-57\%), tronco (13\%-54\%) y con menor frecuencia en la cabeza y el cuello (5\%-33\%). En $10 \%$ a $26 \%$ de los casos, la lesión primaria es oculta. El período observado entre el diagnóstico de melanoma primario y metástasis GI se reporta hasta 54 meses (14). Alrededor de $70 \%$ de los pacientes presenta síntomas relacionados con el tracto GI, mientras que $30 \%$ se mantiene clínicamente asintomático (15).

La TAC es la modalidad preferida de investigación y puede mostrar el compromiso en forma más común como masa intraluminal polipoidea e invaginación intestinal, lesión cavitada o ulcerosa, infiltración difusa e implantes 
(16). La presencia de lesión metastásica se ha confirmado por exploración quirúrgica en el $80 \%$ de los casos, procedimiento endoscópico en $11 \%$ y por biopsia percutánea en $5 \%$ de los pacientes (15).

El melanoma metastásico de origen primario desconocido se presenta en $1 \%-8 \%$ de todos los diagnósticos de melanoma. El diagnóstico se hace después de haber excluido todas las ubicaciones posibles de foco primario, como se realizó con la paciente. Después de haber analizado sus 33 años de experiencia en el tratamiento del melanoma, Savoia y colaboradores observaron que era imposible localizar el foco primario en 88 de 4881 pacientes. En $31(35,3 \%)$ pacientes, la primera presentación clínica fue metástasis en la piel y el tejido subcutáneo, en 38 (43,2\%) pacientes fue compromiso de ganglios linfáticos, mientras que otros 4 pacientes $(4,5 \%)$ presentaron compromiso en ganglios linfáticos y piel. En los 15 pacientes restantes (17\%), el melanoma presentó metástasis viscerales (4).

Es muy difícil diagnosticar melanoma primario del tracto digestivo. Los criterios diagnósticos sugeridos son ausencia de otra ubicación de melanoma y de nevos cutáneos atípicos, lesión localizada solo en el intestino delgado, sin metástasis a distancia y la presencia de un foco primario en la mucosa. Las metástasis en el hígado y el duodeno fueron identificadas en la paciente (Figura 2 y 3 ) después de un análisis detallado de todos los demás lugares posibles del foco primario. Este no se pudo identificar, por tanto, se hizo diagnóstico de melanoma de origen primario desconocido.

Cuando se sospecha metástasis de melanoma en el tracto digestivo se debe llevar a cabo el diagnóstico por imágenes apropiadas. La primera etapa básica de diagnóstico es la ecografía abdominal, este es un examen no invasivo y de bajo costo. Otro examen es la tomografía computarizada, cuya sensibilidad de identificación de metástasis de melanoma en el tracto digestivo es solo de 60\%-70\%. En cada caso, cuando las metástasis en el tracto digestivo se sospechan clínicamente, es necesario llevar a cabo otros exámenes a pesar de TAC negativo. El examen PET/TC garantiza mayor sensibilidad que la TAC en la identificación de metástasis de melanoma y debe llevarse a cabo si está disponible (4). En el reporte de caso, la ecografía y la TAC revelaron metástasis en el tracto digestivo (Figura 1 y 2 ).

\section{CONCLUSIÓN}

El melanoma de origen primario desconocido se presenta en el $1 \%$ al $8 \%$ de todos los melanomas diagnosticados. Por lo general, se diagnostica cuando los pacientes presentan síntomas clínicos en forma de metástasis, por la aparición de complicaciones (hemorragia digestiva, perforación o alteraciones del tránsito intestinal o de la absorción). Es relevante buscar el foco primario antes de realizar un diag- nóstico de melanoma de origen desconocido, para esto es necesario la evaluación integral del paciente, sin embargo, hay que tener en cuenta que en la mayoría de los casos el foco primario no puede ser descrito.

\section{RECOMENDACIONES}

Se recomienda realizar estudios completos de imagenología en pacientes con diagnóstico de melanoma maligno, con el fin de evitar complicaciones subyacentes a la metástasis, de igual manera realizar un diagnóstico oportuno del foco primario, cuando sea posible, para, de esta manera, aclarar si se trata de un melanoma primario o metastásico con primario desconocido.

\section{Conflicto de intereses}

Los autores declaran no tener ningún conflicto de intereses.

\section{Fuente de financiación}

Sin fuente de apoyo financiero.

\section{REFERENCIAS}

1. Cormier JN, Xing Y, Feng L, Huang X, Davidson L, Gershenwald JE, et al. Metastatic melanoma to lymph nodes in patients with unknown primary sites. Cancer. 2006;106(9):2012-20. doi: doi.org/10.1002/cncr.21835.

2. Giuliano AE, Moseley HS, Morton DL. Clinical aspects of unknown primary melanoma. Ann Surg. 1980;191:98104. doi: https://doi.org/10.1097/00000658-19800100000018.

3. AnbariKK,SchuchterLM,BuckyLP,MickR, SynnestvedtM, Guerry D 4th, et al. Melanoma of unknown primary site: presentation, treatment, and prognosis a single institution study. Cancer. 1997;79:1816-21. doi: https://doi.org/10.1002/ (SICI) 1097-0142(19970501) 79:9<1816: :AID CNCR26>3.0.CO;2-\%23.

4. Savoia P, Fava P, Osella-Abate S, Nardò T, Comessatti A, Quaglino P, et al. Melanoma of unknown primary site: A 33-year experience at the Turin Melanoma Centre. Melanoma Res. 2010;20(3):227-32. doi: 10.1097/ CMR.0b013e328333bc04.

5. LiWX, Wei Y, Jiang Y, Liu YL, Ren L, Zhong YS, et al. Primary colonic melanoma presenting as ileocecal intussusception: case report and literature review. World J Gastroenterol. 2014;20(28):9626-30. doi: 10.3748/wjg.v20.i28.9626.

6. Patel RB, Vasava NC, Gandhi MB. Acute small bowel obstruction due to intussusception of malignant amelonatic melanoma of the small intestine. BMJ Case Reports. 2012;2012. doi: 10.1136/bcr-2012-006352.

7. Schuchter LM, Green R, Fraker D. Primary and metastatic diseases in malignant melanoma of the gastrointestinal tract. 
Curr Opin Oncol. 2000; 12:181-5. doi: 10.1097/00001622200003000-00014.

8. Alghamdi S, Omarzai Y. Metastatic melanoma presenting as intussusception in an 80-year-old man: a case report. Case Rep Pathol. 2013;2013:672816. doi: 10.1155/2013/672816.

9. Pozzobon F, Acosta A, Carreño A, Fierro E. Características del melanoma cutáneo primario en el Instituto Nacional de Cancerología 2006-2010. Rev Colomb Cancerol. 2013;17(3):111-8. doi: https://doi.org/10.1016/S01239015(13)70013-1.

10. Desmond RA, Soong S. Epidemiology of malignant melanoma. Surg Clin North Am. 2003;83:1-29. doi: doi. org/10.1016/S0039-6109(02)00092-0.

11. Nova-Villanueva J, Sánchez-Vanegas G, Porras de Quintana L. Cáncer de piel: perfil epidemiológico de un centro de referencia en Colombia 2003-2005. Rev Salud Púb. 2007;9:595-601. doi: https://doi.org/10.1590/S012400642007000400012 .

12. Villegas M, Jaramillo F. Comportamiento clínico, epidemiológico e histológico del melanoma maligno en el
Departamento de Caldas (Colombia). Rev Asoc Colomb Dermatol. 1999;7:192-6.

13. WoollonsA,DerrickEK,PriceML,DarleyCR.Gastrointestinal malignant melanoma. Int J Dermatol. 1997;36:129-31. doi: doi.org/10.1016/S0039-6109(02)00092-0.

14. McDermott VG, Low VH, Keogan MT, et al. Malignant melanoma metastatic to the gastrointestinal tract. Am J Roentgenol. 1996;166:809-13. doi: 10.2214/ ajr.166.4.8610555.

15. Kawashima A, Fishman EK, Kuhlman JE, et al. CT of malignant melanoma: patterns of small bowel and mesenteric involvement. J Comput Assist Tomogr 1991;15:570-4. doi: https://doi.org/10.1097/00004728-199107000-00008.

16. Amersi FF, Terando AM, Goto Y, Scolyer RA, Thompson JF, Tran AN, et al. Activation of CCR9/CCL25 in cutaneous melanoma mediates preferential metastasis to the small intestine. Clin Cancer Res. 2008; 14:638-45. doi: doi. org/10.1158/1078-0432.CCR-07-2025.

17. Blecker D, Abraham S, Furth E, Kochman ML. Melanoma in the gastrointestinal tract. Am J Gastroenterol. 1999;94:342733. doi: 10.1111/j.1572-0241.1999.01604.x. 